\title{
On the Selection of Optimum Routing Protocol in the Presence of Heterogeneous Application Traffic Situations for WSN
}

\author{
Randeep Kaur $^{1}$ and Jyoteesh Malhotra ${ }^{2}$ \\ Department of Computer Science \\ Guru Nanak Dev University, \\ Regional Campus \\ Jalandhar, India. \\ randeep1993@gmail.com, ${ }^{2}$ jyoteesh@gmail.com
}

\begin{abstract}
A network which consists of miniaturized wireless sensor nodes with limited memory capacity and energy source is wireless sensor network. Power consumption is very important in wireless sensor network (WSN) because replacement or recharging of sensor nodes is difficult. There are many routing protocols in wireless sensor network. In this paper, we are considering three routing protocols for different traffic types: Convergecast with WiseRoute, Flooding and ProbabilisticBroadcast. A relative examination of three routing protocols is done by series of simulations in OMNET++. This paper focuses on identifying the best routing protocol on the basis of performance parameters backoff duration, latency, the mean number of hops, the number of frames dropped with interference and number of packets received using uniform, periodic and exponential application traffic situations.
\end{abstract}

Keywords: Wireless sensor network, convergecast, flooding, probabilistic broadcast, Omnet++, backoff, latency, number of hops, application traffic.

\section{Introduction}

Wireless Sensor Network is a distinctive type of Ad-hoc network with a large number of miniature sensor nodes densely deployed in a region to monitor and captures the physical conditions of the environment such as pressure, humidity, temperature etc. the collected environmental data is transmitted to the sink node in a multi-hop manner for further processing. The sensor nodes are also known as motes. They consist of a battery, a limited capacity processing unit and a transceiver. The transceiver is a communication component which consumes the maximum energy [1]. Therefore, we are using IEEE 802.15.4 standard, which is designed for low-rate wireless personal area network. Features of IEEE 802.15.4 are low power consumption, CSMA/CA channel access, Data rates are $240 \mathrm{kbps}, 40 \mathrm{kbps}, 20 \mathrm{kbps}$ [2]. The nodes require more power to transmit data than sensing. Therefore, for the selection of an optimal routing protocol, power management is necessary. The architecture for WSN follows the OSI Model. The architecture of WSNs draws upon many features including signal processing, networking, information managing, and distributed systems [3]. There are three network routing protocols in the MIXIM Framework in Omnet++. Flooding protocol remembers already broadcasted messages and stores them. If it gets another copy of that message it does not rebroadcast them. Convergecast routing protocol uses Wiseroute. Wiseroute is a loop-free routing algorithm. From a central network point, a routing tree is built; designed for sensor network and convergecast traffic. Wiseroute avoids the loops by maximizing the link of all nodes in the network. Probabilistic routing protocol is particularly interesting for mobile networks. It reduces the broadcast storm problem at the risk of some nodes in 
the network are not receiving the packets. It is a multi-hop data dissemination protocol [4]. Comparison of these three routing protocols is done by varying application traffic in all the protocols. Sensor application layer in the wireless sensor network is an efficient layer with low backoff duration and minimum number of frames dropped with interference. The time interval between the two packets in the network can follow distributions: Uniform, Periodic and Exponential. Performance metrics latency, backoff duration, the mean number of hops and number of data packets received are used for evaluating the performance of routing protocols.

\section{Literature Survey}

Alexandru Lavric, Valentin Popa [5] in their research compared the performance of Flooding, Convergecast and Probabilistic Broadcast routing protocols in Omnet++ 4.3 in Mixim Framework in terms of various QoS parameters. According to this research, in terms of latency, highest performance is given by flooding routing protocol followed by

Wiseroute (convergecast) and Probabilistic broadcast. Convergecast algorithm gives the highest performance in terms of a number of packets received by nodes. Hameeza Ahmed, Muhammad Khurram [6] analysed the performance of MAC layer protocols in WSN. Research helps in finding the best MAC layer protocol in terms of highest response time. Performance of IEEE 802.11, CSMA, B-MAC, and IEEE 802.15.4 Mac layer protocols is evaluated in terms of packet delivery ratio and mean latency. According to results highest performance is given by the IEEE 802.11 MAC layer protocol followed by the IEEE 802.15.4, CSMA, B-MAC. Haafizah Rameeza Shaukat, Fazirulhisyam Hashim [7], evaluates the performance of mobile wireless sensor network in Omnet++ for a simple scenario. It discusses the architecture of a mobile node. Parameters of layers, application layer, MAC layer and network layer can be varied according to application requirements. Lovepreet Kaur and Jyoteesh Malhotra [8], analysed the results in Omnet++ for comparison between flooding and convergecast routing algorithms. Wise route algorithm is good for applications which require reliability in terms of less packet loss in the network. Flooding performs better in terms of latency. Mekkaoui Kheireddine, Rahmounr Abdellatif [9], focuses on energy efficiency for enhancing the network's life. It uses optimal hop- length for the retransmission of packets. Jatinder Pal Singh, Love Kumar [10], the optimal value energy consumption in physical layer for IEEE 802.15.4 standard is calculated. Evaluation for convergecast routing shows that for static nodes energy efficiency is $81.2 \%$ more than a network with mobile nodes. S. Kami Makki, Matthew Stangl and Niki Pissinou [11], determined the energy efficient data fusion assurance scheme. CSMA-CA wireless sensor parameters are used in Mixim framework. The wireless sensors used in simulations are: TelosB, Mica2, MicaZ and TI CC1100. Taking network size in consideration for assessment of outcomes, the "sleep/ wakeup scheduling" of silent negative method performs better than other data fusion assurance schemes. Yasmine Derdour, Bouabdellah Kechar, Faycal Khelfi [12], gave simulation based results for the impact on WSN for mobile elements. Results show that the network with relay nodes performs better than the network with a mobile sink.

\section{Simulation Methodology}

In order to understand the issues related with wireless sensor network simulation environment is necessary. For our research we are using Omnet++ Version 4.6 environment with Mixim Framework [4][13]. Omnet++ is C++ programming based objective modular network. Distributed systems and communication networks are simulated with this framework. It is flexible in nature, therefore easy to use for different applications. 
For implementing flooding, wiseroute and probabilistic broadcast routing, 5 sensor nodes are deployed in an area with playground size $600 * 600$. Other simulation parameters for our scenario are given below:

Table 1. Simulation Parameters

\begin{tabular}{|c|l|c|}
\hline Sr. No. & \multicolumn{1}{|c|}{ Parameter } & Value \\
\hline 1. & Number of nodes & 5 \\
\hline 2. & Carrier Frequency & $2.4 \mathrm{GHz}$ \\
\hline 3. & Thermal Noise & $-100 \mathrm{dBm}$ \\
\hline 4. & Header Length & $24 \mathrm{bit}$ \\
\hline 5. & Mobility & Constant Speed \\
\hline 6. & $\begin{array}{l}\text { PMax(Sending } \\
\text { Power) }\end{array}$ & $1.1 \mathrm{~mW}$ \\
\hline 7. & $\begin{array}{l}\text { Route } \\
\text { Interval }\end{array}$ & $1200 \mathrm{~s}$ \\
\hline 8. & Network Type & $\begin{array}{c}\text { Wise route, Flooding, } \\
\text { ProbabilisticBCast }\end{array}$ \\
\hline 9. & RSSI & $-50 \mathrm{dBm}$ \\
\hline 10. & Simulation Time & 5 min \\
\hline 11. & Traffic Type & $\begin{array}{c}\text { Uniform, Periodic, } \\
\text { Exponential }\end{array}$ \\
& & \\
\hline
\end{tabular}

Mixim is a mixed simulator for wireless and mobile sensor networks. User gets a clean interface through Mixim because it hides the complexity of Omnet++ simulations. It has rich tool box. It contains various network and communication protocols. Mixim provides the flooding, convergecast and probabilistic broadcast protocols in network layer. Initial deployment of sensor nodes is given in figure 1 .

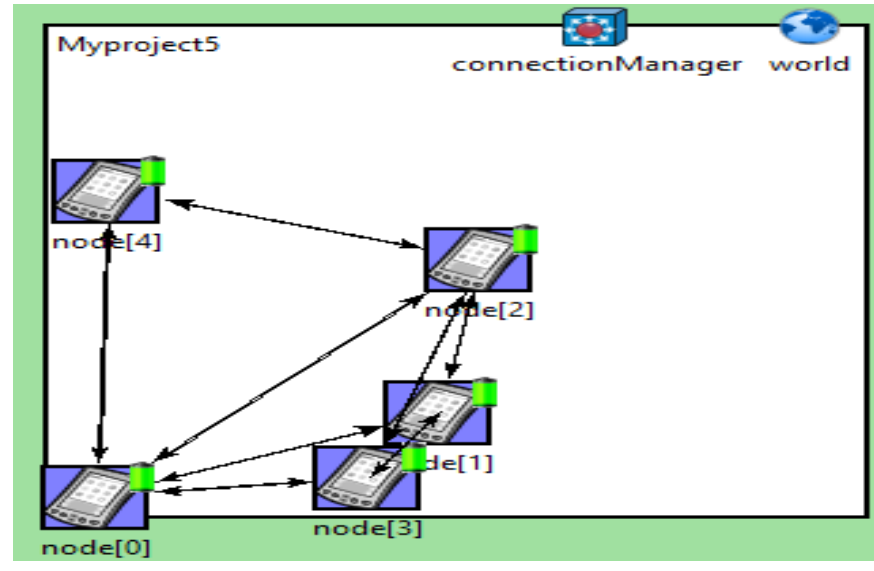

Figure 1. Deployment of Nodes 


\section{Simulation Results}

\subsection{Uniform Traffic Type}

4.1.1. Backoff Duration: Figure 2 shows that ProbabilisticBCast perfoms better than other routing protocols with lowest backoff duration i.e. less no. of retransmissions hence low wastage of energy followed by flooding and convergecast.

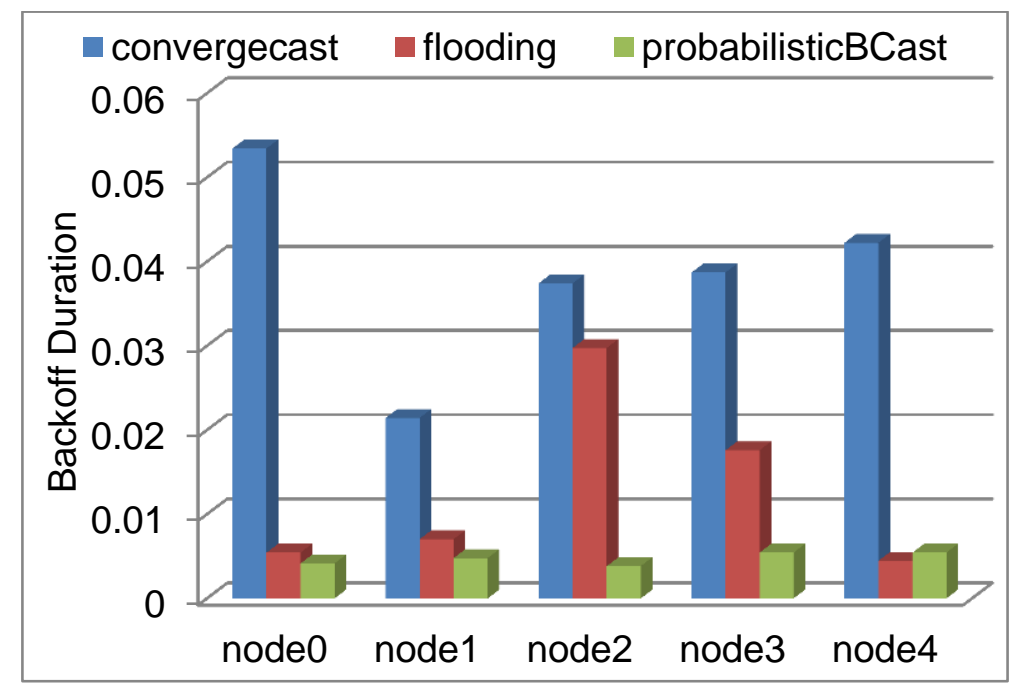

Figure 2. Backoff Duration for Uniform application traffic

4.1.2. Latency: Latency with flooding is less than other two routing protocols. Therefore flooding is used for applications requiring quick response. In probabilistic broadcast latency is quite high i.e. time taken to receive the information is high hence performance is low. Figure 3 shows the results obtained in simulation environment.

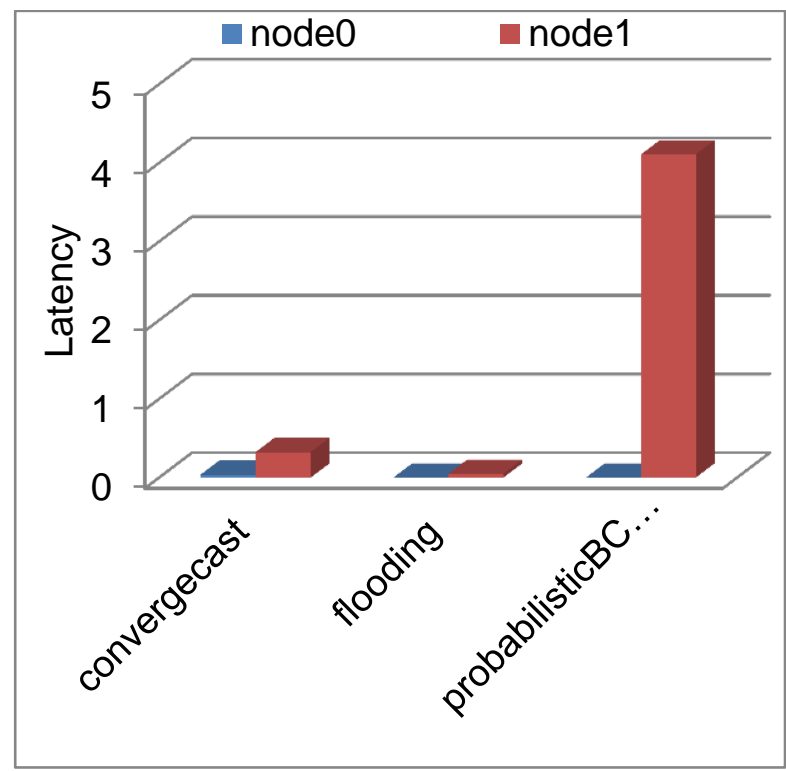

Figure 3. Latency for Uniform Application Traffic 
4.1.3. Mean number of Hops: Hop length influences the energy efficiency of a protocol [8]. Protocol with minimum number of hops is considered more energy efficient. Therefore, according to Figure 4, performance of convergecast is best followed by probabilistic broadcast and flooding.

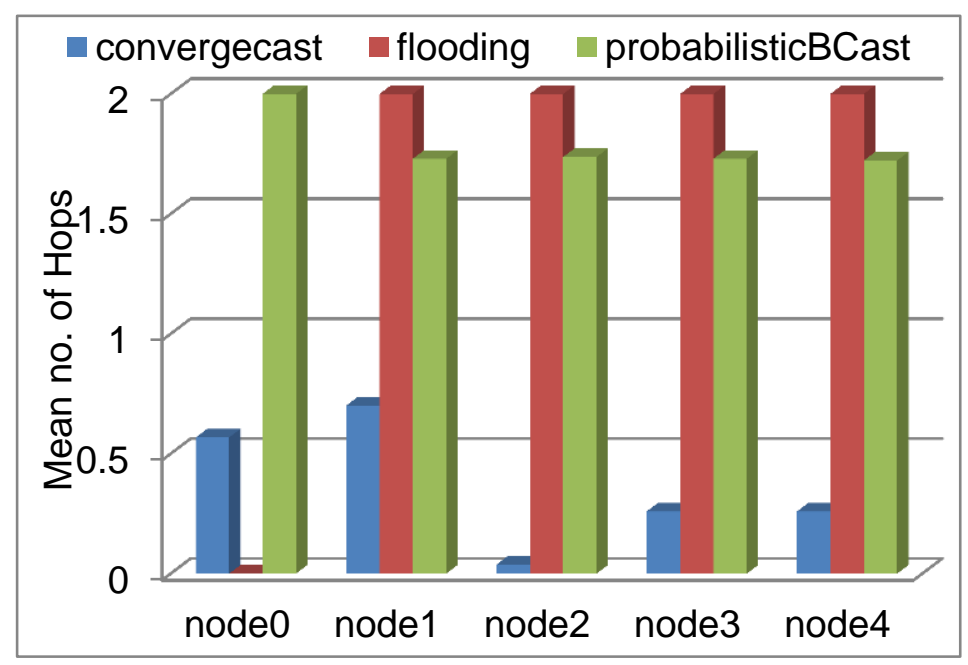

Figure 4. Number of hops for Uniform traffic type

4.1.4. Number of Data Packets Received: Figure 5 shows that maximum number of data packets are received by probabilistic broadcast followed by convergecast and flooding.

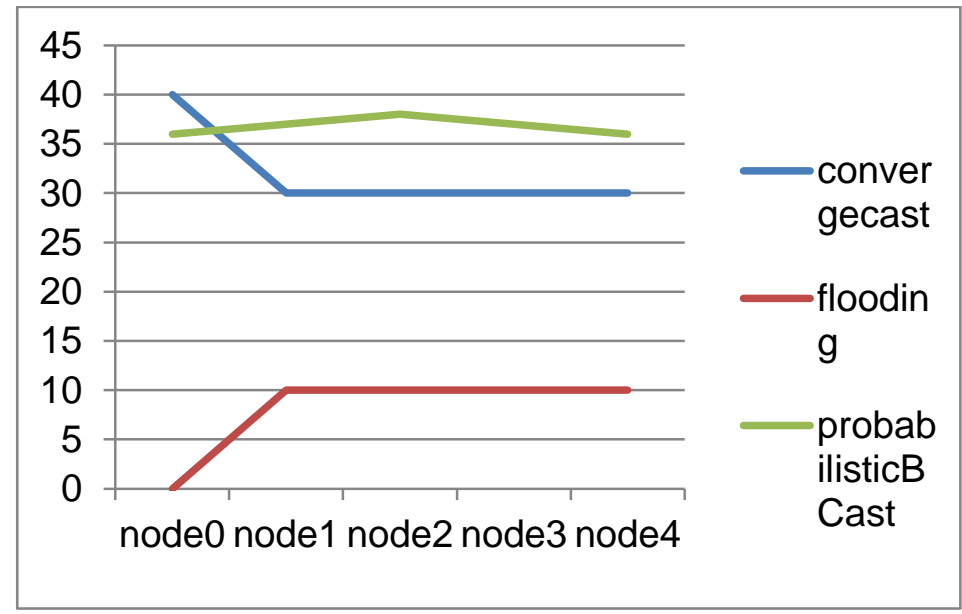

Figure 5. Number of Data Packets Received for Uniform Traffic Type

4.1.5. Number of Frames Dropped with Interference: Figure 6 shows highest performance of Probabilistic broadcast with 0 number of frames dropped with interference. Highest numbers of frames are dropped in convergecast routing. 


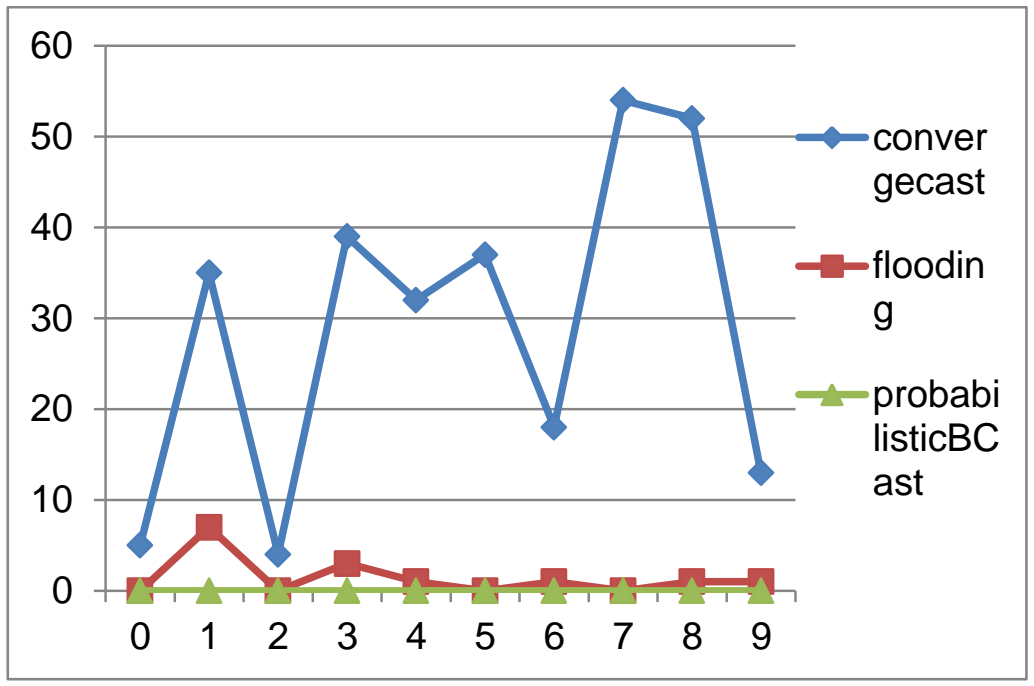

Figure 6. Number of Frames Dropped with Interference in Uniform Traffic Type

\subsection{Periodic Traffic Type}

4.2.1. Backoff Duration: Figure 7 shows minimum backoff duration is given by probabilistic broadcast hence highest performance. Performance of flooding and convergecast is followed by probabilistic broadcast.

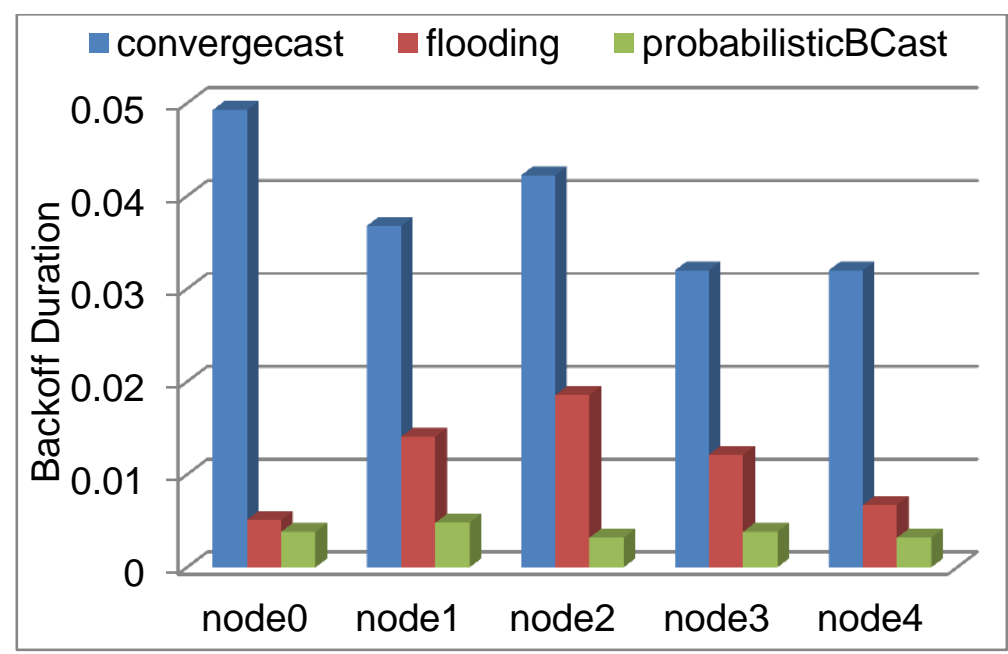

Figure 7. Backoff for Periodic traffic type

4.2.2. Latency: Figure 8 shows lowest latency with flooding hence highest performance followed by convergecast and probabilistic broadcast. 


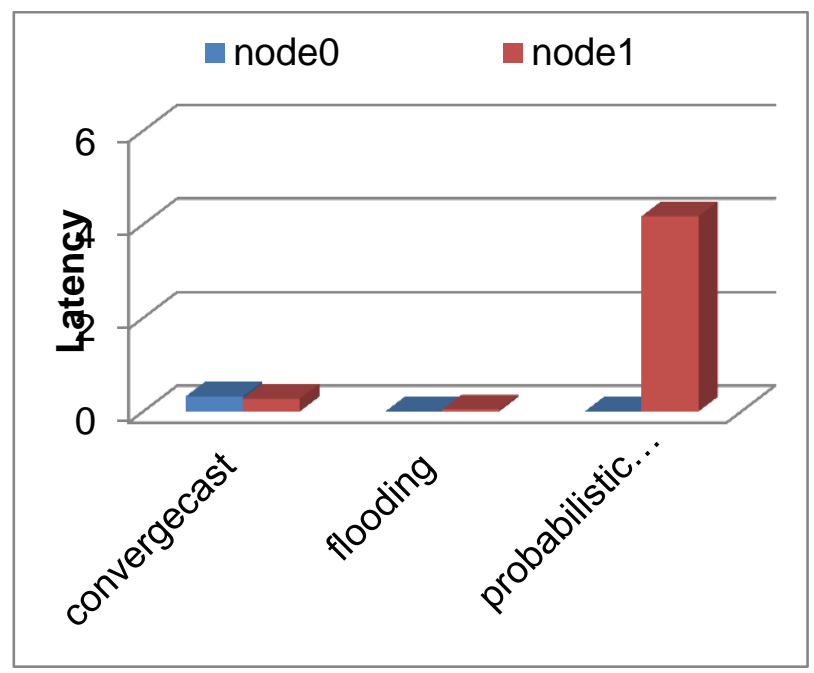

Figure 8. Latency for Periodic Traffic Type

4.2.3. Mean number of hops: Minimum numbers of hops are in convergecast routing protocol. Performance is evaluated with this parameter as, highest performance by convergecast followed by probabilistic broadcast and flooding.

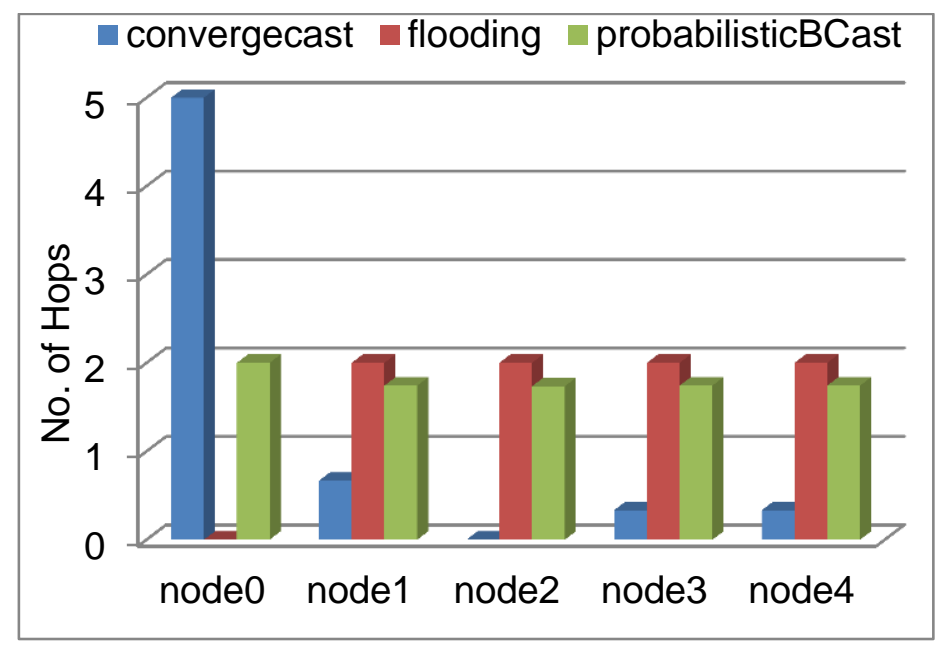

Figure 9. Number of Hops for Periodic Traffic Type

4.2.4. Number of Data Packets Received: Figure 10 shows that maximum numbers of data packets are received by probabilistic broadcast followed by convergecast and flooding. 


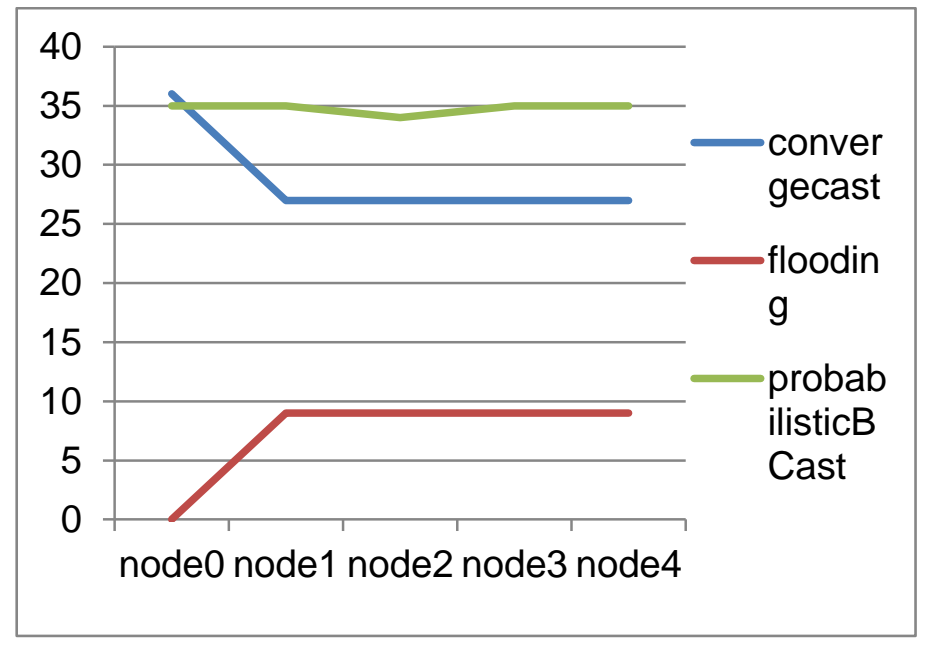

Figure 10. Number of Data Packets Received for Periodic Traffic Type

4.2.5. Number of Frames Dropped with Interference: Figure 11 shows highest performance of Probabilistic broadcast with 0 number of frames dropped with interference. Highest numbers of frames are dropped in convergecast routing.

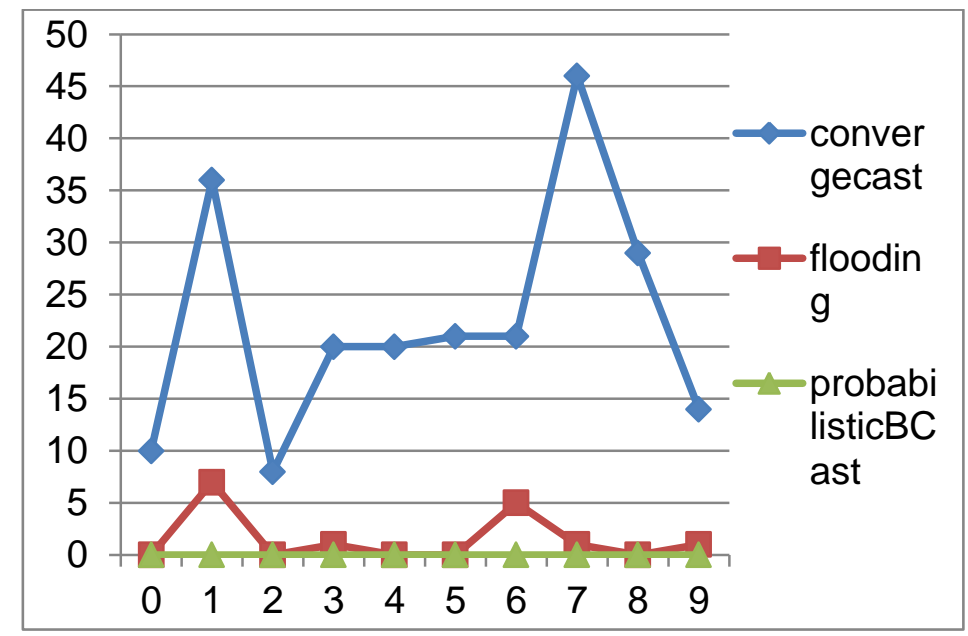

Figure 11. Number of Dropped Frames with Interference

\subsection{Exponential Traffic Type}

4.3.1. Backoff Duration: It denotes the retransmissions. Therefore routing protocol with low value of this parameter shows better performance. In this case, Figure 12 shows low backoff duration for probabilistic broadcast followed by flooding and convergecast. 


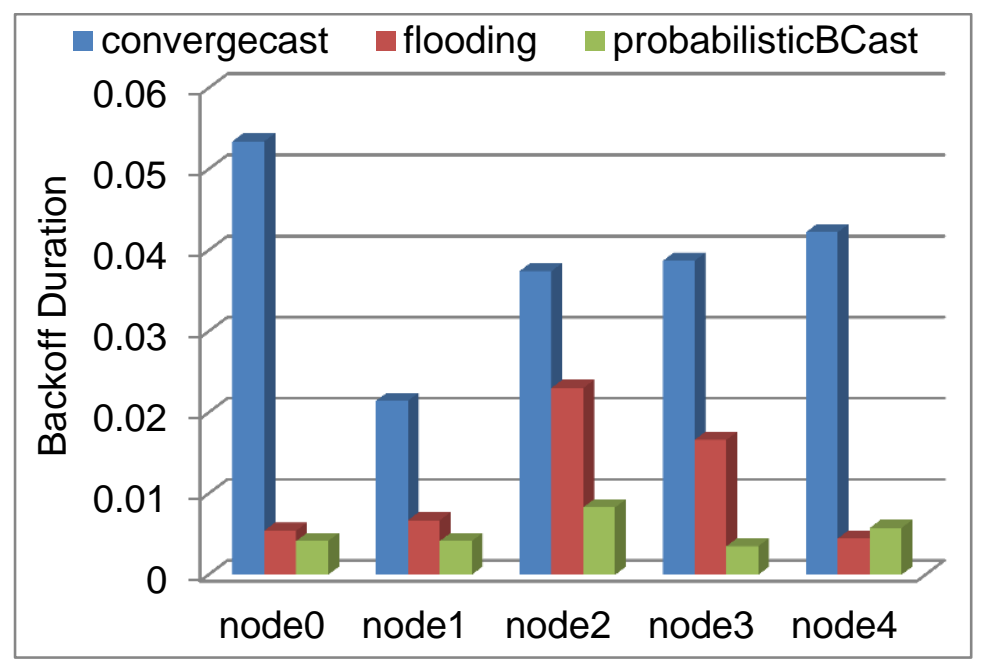

Figure 12. Backoff Duration for Exponential Traffic Type

4.3.2. Latency: Figure 13 shows lowest latency with flooding hence highest performance followed by convergecast and probabilistic broadcast.

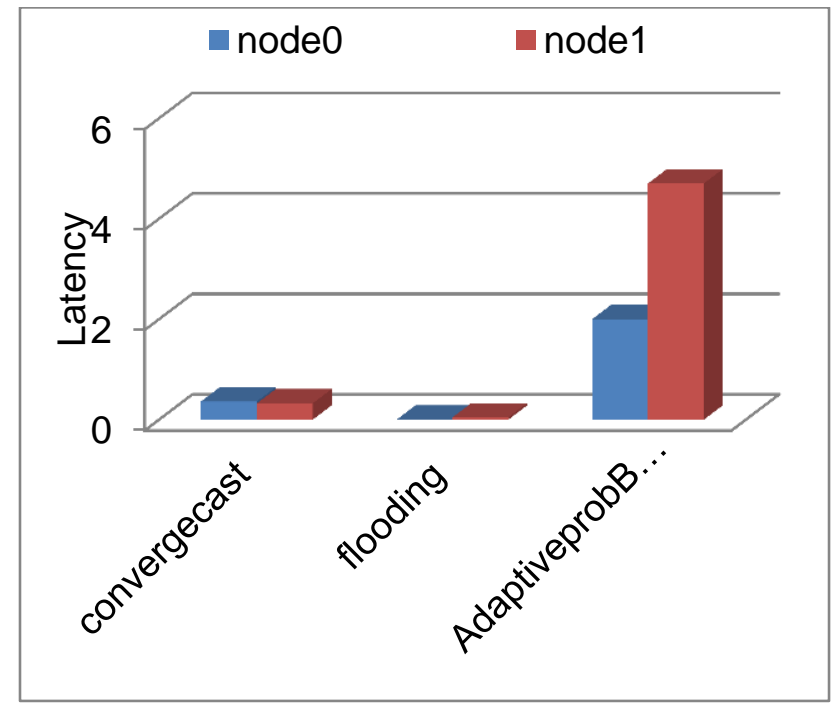

Figure 13. Latency for Exponential Traffic Type

4.3.3. Mean Number of Hops: Protocol with minimum number of hops is considered more energy efficient. Therefore, according to Figure 14, performance of convergecast is best followed by probabilistic broadcast and flooding. 


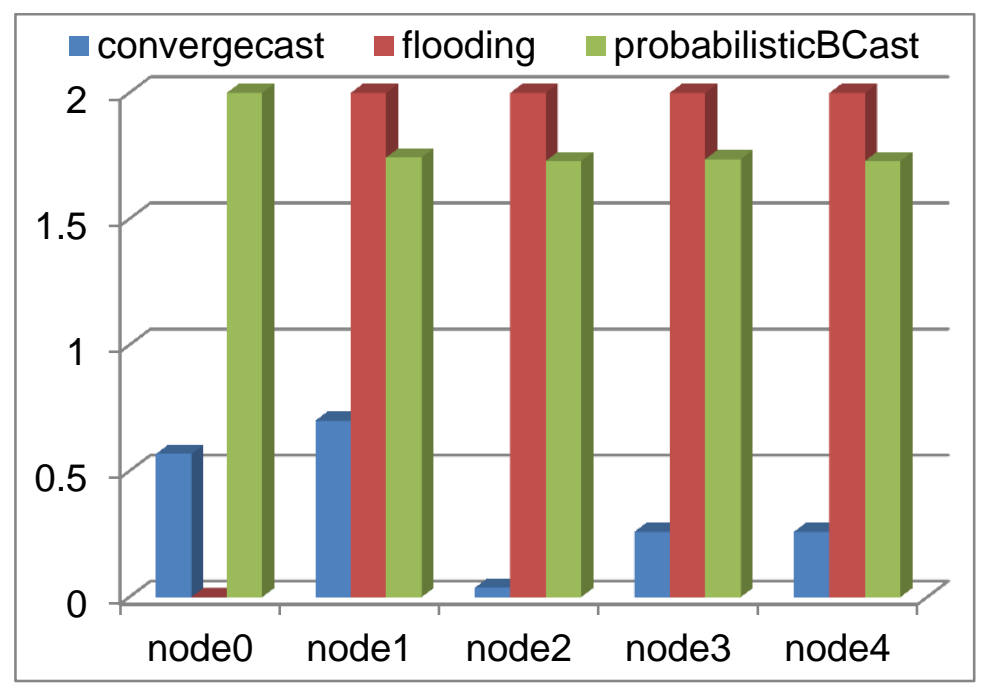

Figure 14. Number of Hops for Exponential Traffic Type

4.3.4. Number of Data Packets Received: Figure 15 shows that maximum numbers of data packets are received by probabilistic broadcast followed by convergecast and flooding.

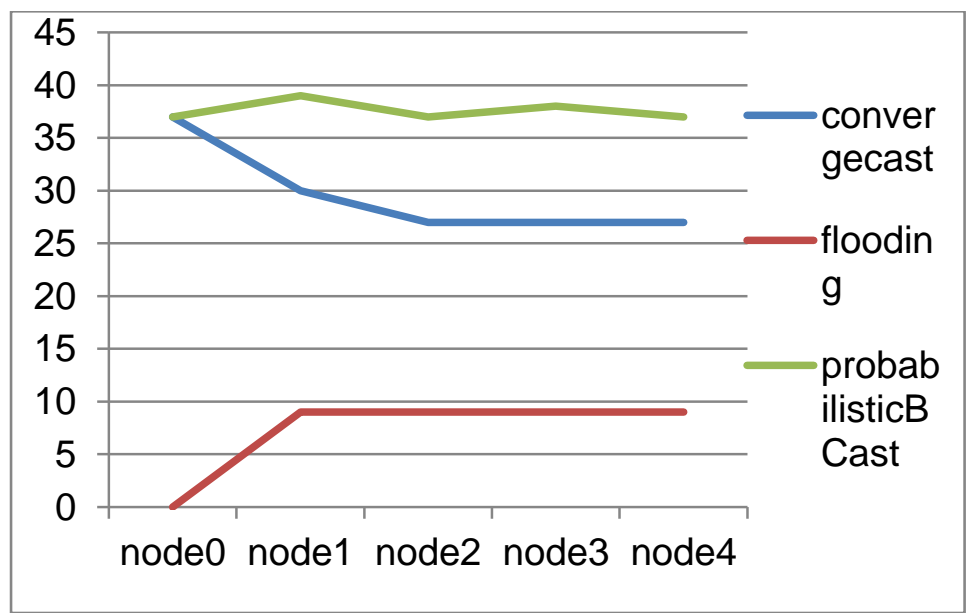

Figure 15. Number of Data Packets Received for Exponential Traffic Type

4.3.5. Number of Frames Dropped with Interference: Figure 16 shows highest performance of Probabilistic broadcast with 0 number of frames dropped with interference. Highest numbers of frames are dropped in convergecast routing. 


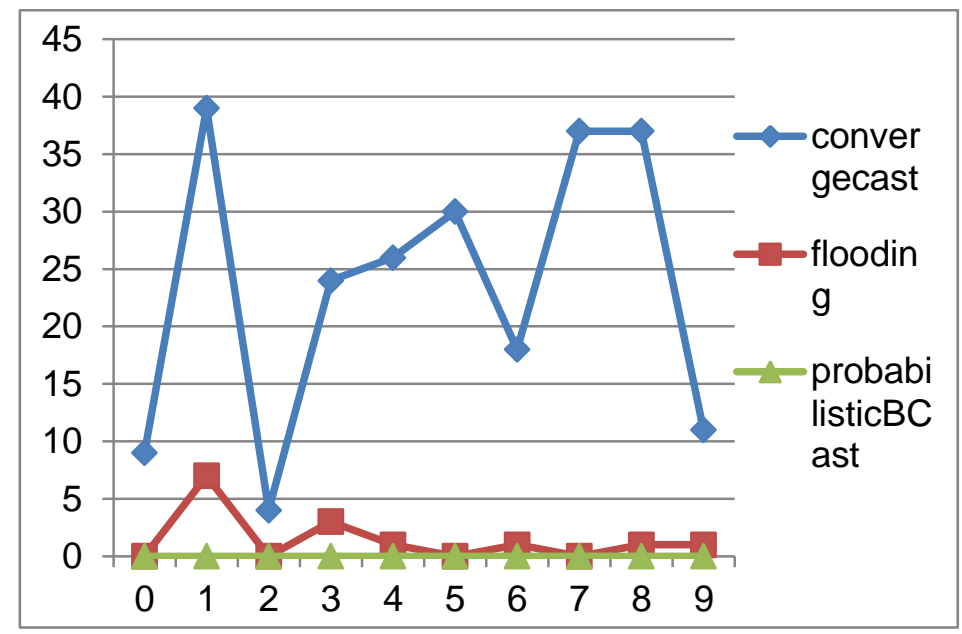

Figure 16. Number of Frames Dropped with Interference for Exponential
Traffic Type

\section{Conclusion}

In this paper, we used three network types Wiseroute, flooding, probabilistic broadcast. A simulation based assessment is done for evaluating the performance of different routing protocols using different application traffic situations in terms of QoS parameters: backoff duration, latency, a mean number of hops, the number of data packets received, the number of frames dropped with interference. Results show that there is a difference between values of uniform, periodic and exponential traffic situations for all the parameters. However, probabilistic broadcast performs much better than convergecast and flooding. In probabilistic broadcast, data is sent with a risk of some nodes not receiving the packets. Nodes receiving the information are selected with some probability. However, the choice of network is application specific. Applications requiring information without loss of packets with interference, low backoff duration (i.e. fast retransmission) and reliable transmission; probabilistic broadcast mechanism is best. If an application requires less numbers of hops for transmission then convergecast is a choice. Choice of traffic pattern has a significant influence on the performance of the model.

\section{Acknowledgement}

First of all I would like to thank Dr. Jyoteesh Malhotra for his continuous support and suggestions throughout the research work. I am thankful to all the staff and faculty members of the department of computer science and engineering. Last but not the least I would like to thank my family members for their continuous motivation and support.

\section{References}

[1] A. Roy and N. Sarma, "Energy Saving in MAC Layer of Wireless Sensor Networks: a Survey", National Workshop in Design and Analysis of Algorithm (NWDAA), Tezpur University, India, (2010).

[2] Available at http://www.ieee802.org/15/pub/TG4.html.

[3] Christo Kleu, "An Ultra-Low Duty Cycle Sleep Scheduling Protocol Stack for Wireless Sensor Network", University of Pretoria, March (2012).

[4] http://www.omnetpp.org/.

[5] Alexandru Lavric, Valentin Popa, "Performance Evaluation of routing algorithms used in largescale WSN: A Step Towards A Smart City", (2015), ISSN 0033-2097.

[6] Hameeza Ahmed, Muhammad Khurram, "Performance Analysis of MAC Layer Protocols in Wireless Sensor Network", I.J. Information Engineering and Electronic Business, Vol. 5, (2014), pp. 44-52. 
[7] Haafizah Rameeza Shaukat, Fazirulhisyam Hashim, "MWSN Modeling Using OMNeT++ Simulator" Fifth International Conference on Intelligent Systems, Modelling and Simulation, IEEE DOI 10.1109, (2014).

[8] Lovepreet Kaur and Jyoteesh Malhotra, "Comparison of Wise Route and Flooding Network Type of Convergecast Routing in Wireless Sensor Network" International Journal of Sensor and Its Applications for Control Systems Vol.3, No.2, (2015), pp.1-10.

[9] Mekkaoui Kheireddine, Rahmoun Abdellatif, "Short-hops vs. Long-hops - Energy efficiency analysis in Wireless Sensor Networks" Vol.6, No. 6, (2014).

[10] Jatinder Pal Singh, Love Kumar, "Power Consumption Analysis of Wireless Sensor Networks with Different Modulation and Routing Techniques", International Journal of Emerging Technologies in Computational and Applied Sciences (IJETCAS),(2014), ISSN Print: 2279-0047.

[11] S. Kami Makki, Matthew Stangl and Niki Pissinou, "Energy consumption in Wireless Sensor Network using Data Fusion Assurance", International Journal of Wireless \& Mobile Networks (IJWMN) Vol. 5, No. 2, April (2013).

[12] Yasmine DERDOUR, Bouabdellah KECHAR, Fayçal KHELFI, "The Impact of the Mobile Element on Performance Improvement in Wireless Sensor Network", The 5th International Conference on Ambient Systems, Networks and Technologies (ANT) (2014).

[13] MIXIM, http://mixim.sourceforge.net/.

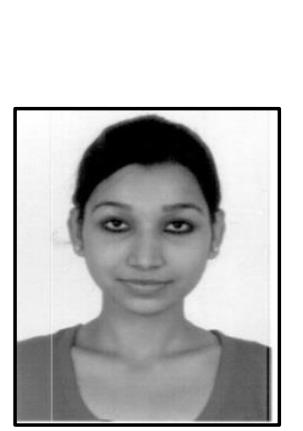

\begin{abstract}
Author
Randeep Kaur, was born in Jalandhar City, State Punjab, on 8 June 1993. She completed B.Tech. from Rayat and Bahra Institute of Engineering \& Bio-Tech., Mohali Campus and M.Tech. from Guru Nanak Dev University Regional Campus, Jalandhar during 2014-2016. Her research interests are in area of Energy-Efficient Routing Protocols for Wireless Sensor Network (WSN). Contact email: randeep1993@gmail.com.
\end{abstract}

Jyoteesh Malhotra was born in Jalandhar, Punjab, INDIA. He completed B.Engg. with Distinction from P.R.M Institute of Technology \& Research, Amravati and M.Tech. with University Gold Medal from Guru Nanak Dev Enginnering College, Ludhiana. He received $\mathrm{PhD}$ in recognition to his contribution in the field of Wireless Communication \& Networks. From 1994 to 2007 he was employed with DAVCMC, New Delhi as Lecturer and Panjab University, Chandigarh as Assistant Professor. He joined Guru Nanak Dev University Regional Campus at Jalandhar in July 2007 where he is currently Professor and Associate Dean (Academics).

His research interests are in the broad area of Pervasive Communication systems and Networks with emphasis on Statistical modeling of Fading Channels, Fading mitgitaion techniques, Optimization of High data rate Optical and wireless Communication Systems, Enhancement of QoS aware Wireless networks and Wireless Security. Dr. Malhotra has published and presented more than 170 technical papers in scientific journals and international conferences and authored 02 books. He is a life member of Indian Society for Technical Education (I.S.T.E.) and Editorial Board of many International Journals of repute. 